\title{
Visualization of gas-liquid mass transfer and wake structure of rising bubbles using pH-sensitive PLIF
}

\author{
M. Stöhr $\cdot$ J. Schanze $\cdot$ A. Khalili
}

Received: 15 June 2008/Revised: 23 December 2008/Accepted: 9 February 2009/Published online: 26 March 2009

(C) The Author(s) 2009. This article is published with open access at Springerlink.com

\begin{abstract}
A planar laser-induced fluorescence (PLIF) technique for visualizing gas-liquid mass transfer and wake structure of rising gas bubbles is described. The method uses an aqueous solution of the $\mathrm{pH}$-sensitive dye Naphthofluorescein and $\mathrm{CO}_{2}$ as a tracer gas. It features a high spatial resolution and frame rates of up to $500 \mathrm{~Hz}$, providing the ability to capture cinematographic image sequences. By steering the laser beam with a set of two programmable scanning mirrors, sequences of three-dimensional LIF images can be recorded. The technique is applied to freely rising bubbles with diameters between 0.5 and $5 \mathrm{~mm}$, which perform rectilinear, oscillatory or irregular motions. The resulting PLIF image sequences reveal the evolution of characteristic patterns in the near and far wake of the bubbles and prove the potential of the technique to provide new and detailed insights into the spatio-temporal dynamics of mass transfer of rising gas bubbles. The image sequences further allow the estimation of bubble size and rise velocity. The analysis of bubble rise velocities in the Naphthofluorescein solution indicates that surfactant-contaminated conditions are encountered.
\end{abstract}

M. Stöhr · J. Schanze · A. Khalili

Max Planck Institute for Marine Microbiology,

Celsiusstr. 1, 28359 Bremen, Germany

\author{
A. Khalili \\ Jacobs University Bremen, Campus Ring 1, \\ 28759 Bremen, Germany \\ e-mail: akhalili@mpi-bremen.de \\ Present Address: \\ M. Stöhr ( $\square)$ \\ German Aerospace Center (DLR), Stuttgart, Germany \\ e-mail: michael.stoehr@dlr.de \\ J. Schanze \\ Massachusetts Institute of Technology, Cambridge, USA \\ e-mail: schanze@mit.edu
}

\section{Introduction}

The fluid dynamics of gas bubbles in liquids has applications in many disciplines, including bubble column reactors in chemical engineering and air-sea gas exchange in geosciences. In particular, the mass transfer between bubbles and liquid often determines the characteristics of the entire system, such as the performance of a reactor or the rates of global chemical cycles. Understanding and quantifying of bubble mass transfer is therefore an important issue in many fields.

The mechanisms of gas-liquid mass transfer have been investigated for several decades in order to estimate gas exchange between ocean and atmosphere (see, e.g., the reviews by Jähne and Haußecker 1998; Turney and Banerjee 2008). In general, the mass transfer between a gaseous and a liquid phase is governed by an interplay between molecular diffusion and convective transport. In a thin film around the interface, the so-called mass boundary layer, transport is restricted to molecular diffusion. Next to this layer, the fluid is renewed continuously or unsteadily by convective transport. The most common concepts, namely surface renewal models and surface divergence models, essentially state that the dynamics of the renewal is most important for the rate of mass transfer. The mass transfer rate of a rising bubble thus depends strongly on the convective transport in the near wake.

Depending on its size, a freely rising bubble may perform a rectilinear, zigzagging, spiralling or rocking motion (Clift et al. 1978; Magnaudet and Eames 2000). Each case is associated with characteristic vortex structures in the near wake of the bubble, and it is therefore expected that the mechanisms of mass transfer vary significantly for the different path instabilities. Furthermore there are considerable differences in the fluid dynamics at the interface 
between pure water and water that is contaminated with surfactants (Bel Fdhila and Duineveld 1996).

The flow field in the wake of rising bubbles has been studied using particle image velocimetry (Brücker 1999; Lindken and Merzkirch 2000; Fujiwara et al. 2004), laser doppler anemometry (Ellingsen and Risso 2001), dye visualization (Lunde and Perkins 1997) and schlieren optics (de Vries et al. 2002). While these studies primarily aimed to improve the understanding of the path instabilities, the detailed mechanisms of mass transfer remain largely unclear because the spatio-temporal dynamics in the close vicinity of the bubble, i.e., the boundary layer at the interface, could not be sufficiently resolved.

As a complementary approach, planar laser-induced fluorescence (PLIF) has been widely used for the direct twodimensional visualization of scalar transport in aqueous flows (Crimaldi 2008). The usage of LIF for the study of gas-liquid mass transfer requires a suitable combination of a watersoluble gas and a fluorescent dye that is sensitive to the gas. Methods using oxygen quenching of pyrenebutyric acid or the $\mathrm{pH}$-sensitivity of fluorescein in combination with $\mathrm{HCl}$ gas have been employed for the study of mass transfer at free or wind-forced air-water interfaces in order to quantify gas exchange of oceans or rivers (Münsterer and Jähne 1998; Herlina and Jirka 2004; Walker and Peirson 2008).

The aim of the present study was the development of a PLIF technique that allows for the qualitative visualization of gasliquid mass transfer at the interface and wake of rising gas bubbles. The method uses PLIF of the $\mathrm{pH}$-sensitive dye Naphthofluorescein with carbon dioxide $\left(\mathrm{CO}_{2}\right)$ as a tracer gas. It features a high spatial resolution and a frame rate of up to $500 \mathrm{~Hz}$, which provides the ability to capture cinematographic image sequences. Using a set of two programmable scanning mirrors for steering of the laser beam, the field of view can be easily adapted to the scale of interest and scanned in the out-of-plane direction. The latter allows for recording sequences of three-dimensional LIF images, similar to studies of, e.g., Deusch and Dracos (2001) and Tian and Roberts (2003). A series of experiments on rising bubbles with diameters between 0.5 and $5 \mathrm{~mm}$ demonstrates that the technique provides detailed insights into the spatio-temporal dynamics of mass transfer.

\section{Experimental method}

\subsection{PLIF apparatus and water tank}

The technique for visualization of gas-liquid mass transfer is based on the change of $\mathrm{pH}$ of an aqueous solution resulting from the dissolution of $\mathrm{CO}_{2}$. The change of $\mathrm{pH}$ is imaged using PLIF of a pH-sensitive dye. The experimental setup of the PLIF technique is shown in Fig. 1. The water is contained in a

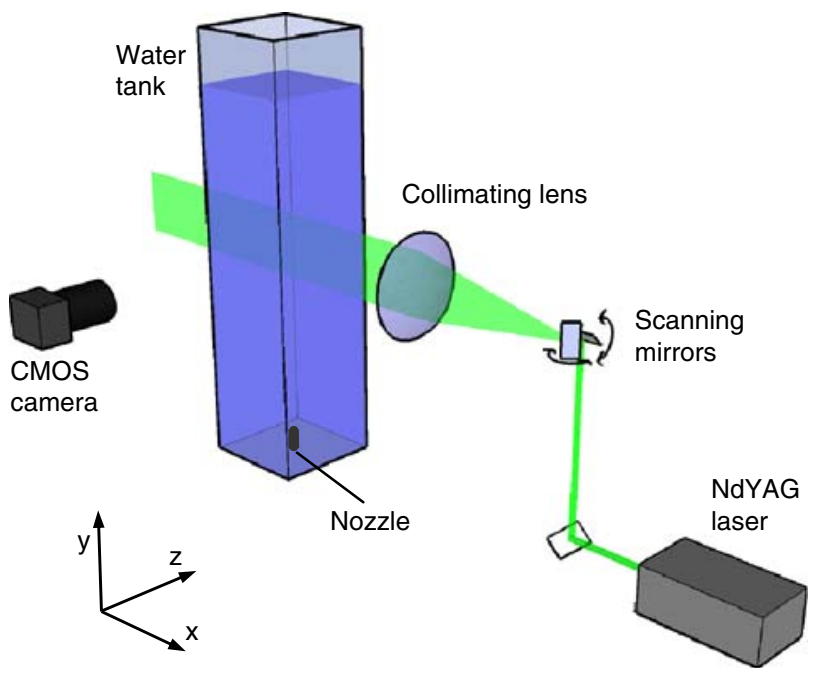

Fig. 1 Experimental setup

box-shaped tank with a square cross section of $200 \mathrm{~mm}$ side length and a height of $800 \mathrm{~mm}$. The side walls are made of transparent glass to provide optical access for laser and camera. An electronic $\mathrm{pH}$ sensor is attached at the top of the tank to continuously monitor the acidity of the water. Bubbles are generated by a syringe with a needle of $0.5 \mathrm{~mm}$ inner diameter. A small amount of $\mathrm{CO}_{2}$ is pushed manually from the syringe into a vertical tube below the tank and enters the tank at the center of the bottom through a nozzle of $2 \mathrm{~mm}$ inner diameter. The exit of the nozzle is defined as the origin of the coordinate system, $x=y=z=0$.

A diode-pumped frequency-doubled Nd:YAG laser with a continuous wave output of $1.5 \mathrm{~W}$ at $532 \mathrm{~nm}$ is used for the excitation of the fluorescent dye. The beam with a diameter of $1.5 \mathrm{~mm}$ is steered into the tank by two orthogonal mirrors of a dual-axis galvanometer scanner (GSI Lumonics VM 1000). With the two scanning mirrors, the beam is swept across a certain range of the tank in the vertical $(y)$ and horizontal $(z)$ direction. The mirrors are located at the focal point of a plano-convex spherical lens (250 $\mathrm{mm}$ diameter and $1,000 \mathrm{~mm}$ focal length) so that the beam always passes the tank in the horizontal $(x)$ direction. The positions of the scanning mirrors are controlled by external voltage signals, which are generated by a programmable control unit (PCU).

Both planar (2D) and volumetric (3D) LIF image sequences were captured using different paths of the laser beam in the $y z$ plane. In the case of $2 \mathrm{D}$ measurements, the beam is repeatedly swept up and down, thus forming a vertical plane (Fig. 2a). During each sweep a 2D image is recorded with a CMOS camera focused on this plane. For the $3 \mathrm{D}$ measurements, a series of adjacent $2 \mathrm{D}$ images is recorded by displacing the beam in $z$-direction after each vertical sweep (Fig. 2b). The camera optics remains fixed since the depth of field covered the whole range in the out- 

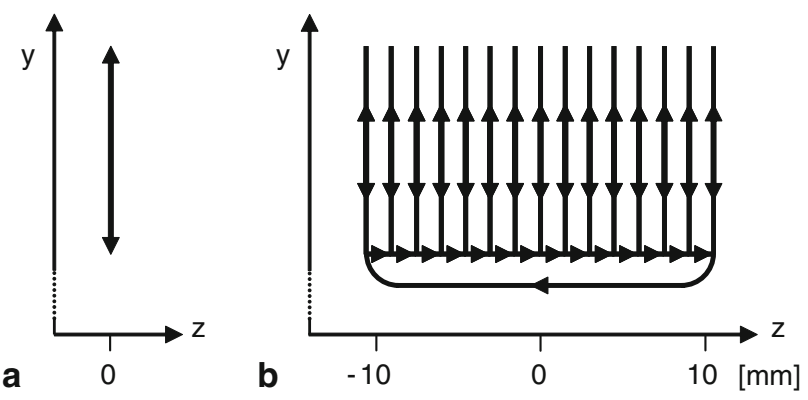

Fig. 2 Paths of laser beam in the $y z$ plane for a 2D and $\mathbf{b} 3 \mathrm{D}$ PLIF experiments

of-plane direction. After each series, corresponding to one $3 \mathrm{D}$ image, the beam is returned to the initial position and the next 3D image is recorded. The 3D images consisted of $152 \mathrm{D}$ images taken over a distance of $21 \mathrm{~mm}$ in $z$-direction, corresponding to a spacing of $1.5 \mathrm{~mm}$.

The CMOS camera is a Basler A504k with a resolution of $1,280 \times 1,024$ pixel, a dynamic range of $8 \mathrm{bit}$, and a maximum frame rate at full resolution of $f=500 \mathrm{~Hz}$. By choosing a smaller resolution (region of interest), higher frame rates can be obtained. The image data is transfered in real-time through a high-speed interface (CameraLink, up to $680 \mathrm{MB} / \mathrm{s}$ ) to the memory of a PC and subsequently written to a hard disk. The camera is equipped with a zoom lens of F2.8 aperture and $28-70 \mathrm{~mm}$ focal length, and an optical bandpass filter with a transmission range between 610 and $690 \mathrm{~nm}$ to let pass only the fluorescent emission and block out scattered laser light. Frame rate, field of view and exposure time of the 2D and 3D LIF imaging are adjusted through the synchronized control of scanning mirrors and camera by the PCU of the galvanometer scanner. At the beginning and end of each vertical sweep the PCU sends a trigger pulse to the external trigger input of the camera to start and stop, respectively, the exposure of the CMOS sensor. Measurements were performed with different fields of view in the $x y$ plane, ranging from $30 \times 23 \mathrm{~mm}^{2}(720 \times 512$ pixel $)$ to $145 \times 210 \mathrm{~mm}^{2}$ $(720 \times 1,024$ pixel). For the present application, the maximum frame rate is not limited by the rate of data transfer, but by the need to acquire enough LIF intensity during the exposure time. It was found that a sufficient signal-to-noise ratio of the images is obtained for exposure times longer than $2 \mathrm{~ms}$. Frame rates of $2 \mathrm{D}$ images were therefore chosen in the range between 250 and $500 \mathrm{~Hz}$, and accordingly between 16.7 and $33.3 \mathrm{~Hz}$ for 3D images.

Because the individual 3D images are composed of consecutively recorded 2D images, they are a good approximation of the instantaneous distribution of concentration only when the frame rate is high enough with respect to the spatio-temporal scales of bubble dynamics. This is, roughly estimated, the case when the displacement of a bubble between two images is not greater than its diameter.
With a typical diameter of $d=3 \mathrm{~mm}$ and a maximum rise velocity of $v=300 \mathrm{~mm} / \mathrm{s}$ this requirement is fulfilled for frame rates $f=v / d>100 \mathrm{~Hz}$. While the frame rates of 2D image sequences are well above this limit, the 3D images thus do not approximate instantaneous concentration fields in the region around the bubble. However, liquid velocities in the wake behind a bubble quickly decay by an order of magnitude (Ellingsen and Risso 2001). The resulting limit of $f=10 \mathrm{~Hz}$ is then fulfilled for the 3D images, and they are consequently considered valid approximations of instantaneous concentration fields in the wake region several diameters behind a bubble.

\section{$2.2 \mathrm{pH}$-sensitive fluorescence}

The tracer of mass transfer is $\mathrm{CO}_{2}$, which has a solubility in water of $7.110^{-4}$ (mole fraction at $p=1$ bar and $T=293 \mathrm{~K}$, Lide 2000), a diffusivity of $D=1.76$ $\times 10^{-5} \mathrm{~cm}^{2} / \mathrm{s}$ (Tamimi et al. 1994) and a Schmidt number $S c \approx 550$. The effect on $\mathrm{pH}$ results from its role as the anhydride of carbonic acid $\mathrm{H}_{2} \mathrm{CO}_{3}$.

Naphthofluorescein has been chosen as the fluorescent dye because it is strongly sensitive to $\mathrm{pH}$ changes near the neutral region $(\mathrm{pH}=7)$ and its excitation spectrum is compatible with the wavelength of a frequency-doubled $\mathrm{Nd}$ :YAG laser $(\lambda=532 \mathrm{~nm})$. For $\mathrm{pH}=9$ the wavelengths of maximum absorption and emission are $\lambda_{\mathrm{abs}}=594 \mathrm{~nm}$ and $\lambda_{\mathrm{em}}=663 \mathrm{~nm}$, respectively (Sigma-Aldrich 2008). Due to the large separation between $\lambda_{\mathrm{abs}}$ and $\lambda_{\mathrm{em}}$, reabsorption of the emitted light is mostly inhibited. When excited at $532 \mathrm{~nm}$ and $\mathrm{pH} \approx 7$, the fluorescent emission was found to be mainly in the range of $600-700 \mathrm{~nm}$.

Naphthofluorescein is soluble in water at $\mathrm{pH}>8$ (SigmaAldrich 2008), and therefore $\mathrm{NaOH}$ was added to the water until a $\mathrm{pH} \approx 8$ was reached before Naphthofluorescein was dissolved. The $\mathrm{pH}$ of the resulting solution was maintained above 6 as Naphthofluorescein tends to flocculate if left at greater acidity for periods of hours. We found that the intensity of fluorescent emission is most sensitive to variations of $\mathrm{pH}$ at $7<\mathrm{pH}<7.5$, and therefore the $\mathrm{pH}$ was kept in this range during the experiments. At this condition the intensity of fluorescence drops abruptly from its value near maximum at $\mathrm{pH} \approx 7$ to virtually zero when acidity changes slightly to $\mathrm{pH} \approx 6$. This implies, however, that any changes in acidity in the range of $\mathrm{pH}<6$ can no longer be detected. For quantitative studies it would therefore be necessary to ensure $\mathrm{pH}>6$ everywhere in the water by choosing an adequate composition of gas in the bubbles. The use of pure $\mathrm{CO}_{2}$ in the present study leads to local regions of $\mathrm{pH}<6$, but in return it gives rise to an overall high sensitivity. We note that the study aims at the visualization of spatio-temporal dynamics of mass transfer, and not at quantitative estimates of concentration or transfer rate. 
The water tank was cleaned with isopropanol and filled with deionized water to a height of $700 \mathrm{~mm}$. A concentration of $\approx 2 \times 10^{-6} \mathrm{M}$ Naphthofluorescein was used, as this produced a sufficient fluorescence in combination with moderate absorption. Higher concentrations lead to increased absorption and thus to a significant decrease of laser light intensity along the laser beam. Before each experiment, the solution was thoroughly stirred in order to ensure the homogeneous distributions of $\mathrm{pH}$ and fluorescent dye. The PLIF measurement is then started after $5 \mathrm{~min}$ of letting the water come to rest.

It is well known that in contaminated water, i.e., water that contains surfactants above a certain concentration, the fluid dynamics at the interface of a bubble is modified and rise velocities are considerably lower (Clift et al. 1978). Due to the essential role of the fluorescent dye, the present PLIF technique does not allow for a study of bubbles in pure water. In order to characterize the contamination of the Naphthofluorescein solution, bubble sizes and rise velocities were determined from a series of PLIF image sequences as described in Sect. 3. Furthermore, a second series of experiments was performed in which $2 \times 10^{-6} \mathrm{M}$ of the soluble surfactant Triton X-100 was added to the water, raising the concentration of surfactants above the critical level for contamination (Bel Fdhila and Duineveld 1996). We discuss the results in Sect. 4.1.

\section{Image analysis}

\subsection{Image preprocessing}

The raw PLIF images contain so-called fixed pattern noise, which is a static pattern superimposed on every image and originates from the electronics of the CMOS sensor. As a first pre-processing step, the fixed pattern noise is therefore removed by subtraction of a dark image that was recorded separately. Furthermore all PLIF images are normalized with respect to nonuniform vertical distribution of laser intensity using the vertical distribution of fluorescence in areas of uniform $\mathrm{pH}$ to the left and right of the bubble trajectory.

\subsection{Determination of rise velocity and bubble size}

Figure 3 a shows examples of a $2 \mathrm{D}$ image sequence performed with a frame rate of $500 \mathrm{~Hz}$. The time interval between the images is $\Delta t=30 \mathrm{~ms}$, i.e., every fifteenth image of the original sequence is shown. The time when the bubble appears at the lower boundary of the laser sheet is defined as $t=0$. The images show regions of low fluorescence intensity (dark gray and black) in the wake of the bubble indicating that $\mathrm{CO}_{2}$ dissolved from the gas phase into the aqueous solution. Black areas appear to the left of the bubble when it shadows the laser beam. The contours of the bubble (white) are visible in the images due to scattering and reflection of fluorescent emissions. This is used for the determination of position and size of the bubble, which is accomplished by fitting an elliptical curve to the bubble contour using a least-squares minimization. The center of the ellipse describes the path of the bubble in the $x y$ plane, $x(t)$ and $y(t)$. The rise velocity $v$ is then determined from the temporal evolution of vertical position, $y(t)$, by means of a straight-line fit. The uncertainty of the rise velocity is estimated as $\Delta v / v=1 \%$.

For the determination of bubble size, an image is chosen where the bubble crosses the plane of the laser sheet, such as at $t=78 \mathrm{~ms}$ in the given example. Assuming an oblate
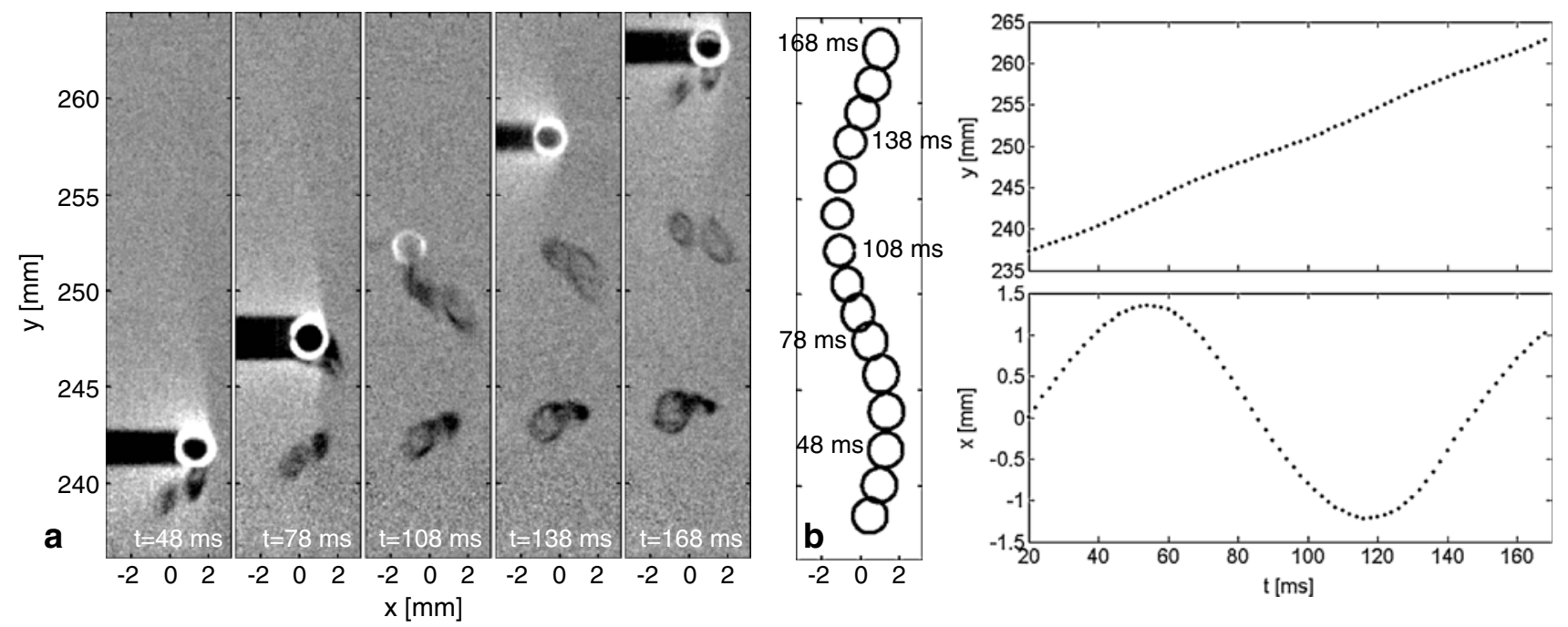

Fig. 3 a Sequence of PLIF images. b Ellipses representing the bubble contour. c Time-series of bubble position 
ellipsoidal shape, the volume of the bubble is determined from the ellipses' major axis $a$ and minor axis $b$ as $V=4 \pi a^{2} b / 3$. The commonly used equivalent spherical diameter is calculated as $d=(6 V / \pi)^{1 / 3}$. Provided that the bubble has a spherical or ellipsoidal shape, the uncertainty of $d$ (resulting from the thickness of the white contour line) is estimated as $\Delta d / d=10 \%$. When the shape differs strongly from an ellipsoid $(d>5 \mathrm{~mm})$, a reliable estimation of size is no longer viable. It is noted that the determination of bubble size is rather a by-product than the main purpose of the PLIF technique, offering an easy approach with reasonable accuracy.

For the present example, Fig. 3b shows the fitted ellipse for every fifth image $(\Delta t=10 \mathrm{~ms})$. The path of the center of the ellipse is plotted in Fig. 3c. The bubble simultaneously oscillates in lateral direction $(-1.2 \mathrm{~mm}<x$ $<1.3 \mathrm{~mm}$ ) and in the out-of-plane direction and crosses the plane of the laser sheet $(z=0)$ at $t=78 \mathrm{~ms}$. The rise velocity is calculated as $v=173 \mathrm{~mm} / \mathrm{s}$, and the bubble size is estimated from the image at $t=78 \mathrm{~ms}$ as $d=2.2 \mathrm{~mm}$.

\section{Results and discussion}

\subsection{Rise velocities}

A first series of 70 PLIF experiments has been performed with deionized water and fluorescent dye, and a second series of 20 experiments with additional Triton X-100. For each experiment bubble size and rise velocity were determined as described in Sect. 3. In this section the results are presented and discussed with regard to contamination of the water and attainment of terminal velocity.

Even small concentrations of solutes in water (e.g., surfactants) can strongly affect the fluid dynamics at the interface of a bubble. For a certain bubble diameter, the free rise velocity is highest in pure water and at a certain critical concentration, it decreases rapidly to a lower limit value. For a number of surfactants including Triton X-100, Bel Fdhila and Duineveld (1996) have shown that this value does not depend on the type of the surfactant and that it is unaffected by further increases in the concentration. The concentration of Triton X-100 used in the second series of experiments $\left(2 \times 10^{-6} \mathrm{M}\right)$ is above the critical concentration specified as $\approx 1 \times 10^{-6} \mathrm{M}$. Figure 4 shows the rise velocities for the two series of experiments along with literature values for clean bubbles (Clift et al. 1978), solid spheres (Clift et al. 1978) and contaminated bubbles (Bel Fdhila and Duineveld 1996). The equivalent diameter of the bubbles is in the range of 0.5 to $5 \mathrm{~mm}$. For $d$ $\leq 1.4 \mathrm{~mm}$ contaminated bubbles mostly behave like solid spheres, whereas for $d>1.4 \mathrm{~mm}$ their shape becomes nonspherical. Oscillations of shape and path start to occur, and

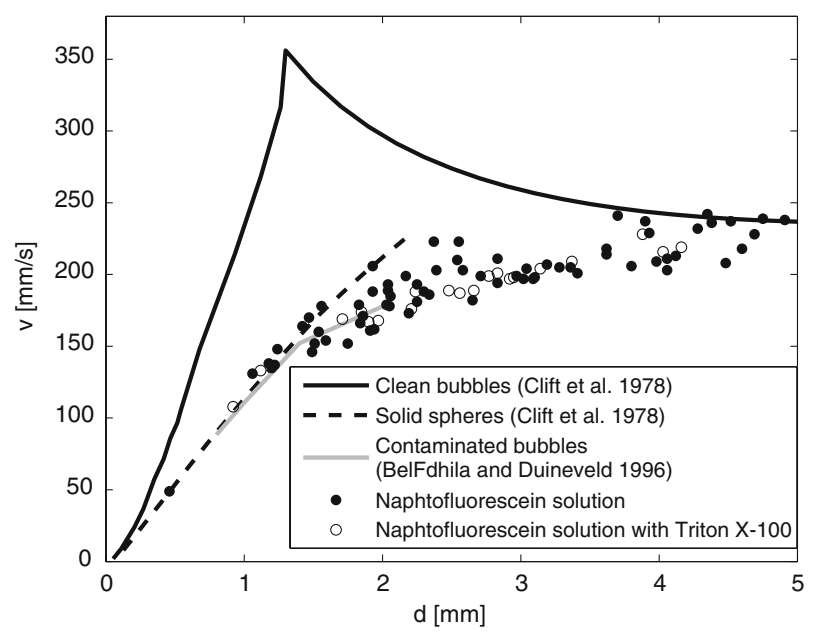

Fig. 4 Literature values and experimental results for bubble rise velocities

rise velocities fall below those of solid spheres. As expected, the experiments with Triton X-100 closely follow the literature values for contaminated bubbles. For the Naphtofluorescein solution without Triton X-100, the values also primarily follow those of contaminated bubbles, but the spread of velocities is larger. Consequently, both cases are considered as essentially contaminated, while the conditions are more accurately defined for the case with Triton X-100.

Bubble rise velocities become stationary only after a certain distance travelled from the location of release, which is different for clear and contaminated conditions and depends on the technique of bubble generation (Zhang and Finch 2001). In the present study, the lowest position where PLIF images of bubbles were recorded was $100 \mathrm{~mm}$ above the nozzle, and the evolution of vertical position of the bubbles in the image sequences showed that stationary rise velocities were attained for all experiments. This agrees with Bel Fdhila and Duineveld (1996), who found that stationary velocities are obtained at $20-40 \mathrm{~ms}$ after release, corresponding to a distance of less than $10 \mathrm{~mm}$.

\subsection{Wake structures and mass transfer}

Preliminary results based on five selected PLIF measurements showing the gas-liquid mass transfer of rising bubbles were obtained. We mainly intend to demonstrate the suitability and potentials of the experimental technique, and thus restrict the discussion of the observed phenomena to a brief description. The specified frame rates denote those of the original PLIF sequences, while often only a subset of the images is shown in the figures. Areas of uniform fluorescence to the left and right of the bubble trajectories have been cut off. The figure captions specify whether Triton X-100 was added or not. 


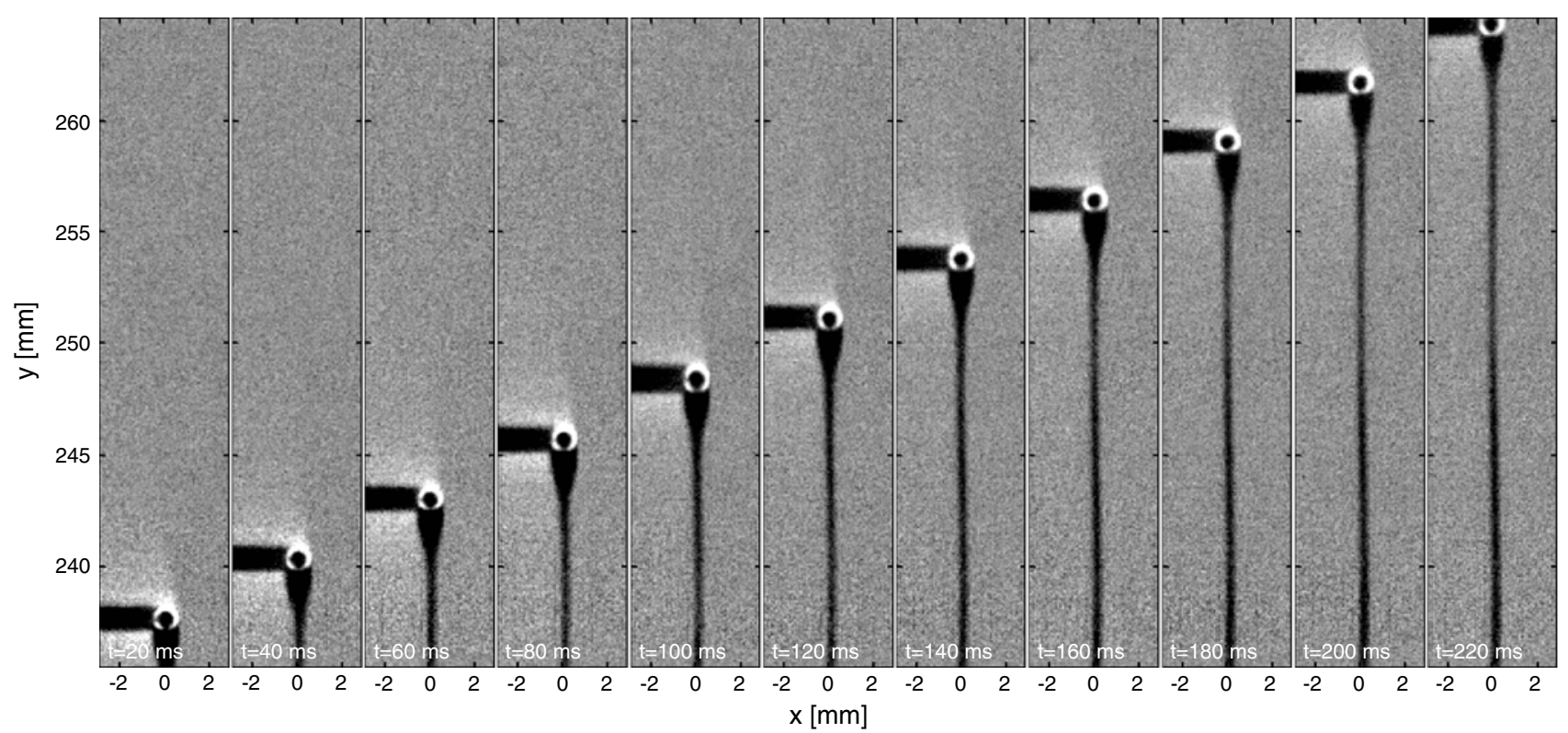

Fig. 5 Sequence of 2D PLIF images showing a bubble with diameter $d=1.1 \mathrm{~mm}$ and rise velocity $v=133 \mathrm{~mm} / \mathrm{s}$ (with Triton X-100)

The first image sequence shown in Fig. 5 was recorded with a frame rate of $f=500 \mathrm{~Hz}$. The bubble with diameter $d=1.1 \mathrm{~mm}$ rises rectilinearly with a velocity of $v=133 \mathrm{~mm} / \mathrm{s}$. It can be seen that $\mathrm{CO}_{2}$ is transferred from the gas phase into the water and accumulates in a region with a length of $\approx 1.5 d$ directly behind the bubble. This indicates that recirculation takes place in the wake of the bubble. The recirculation of dissolved $\mathrm{CO}_{2}$ towards the bubble indicates a reduced surface renewal and thus a low rate of mass transfer. Behind this region, dissolved $\mathrm{CO}_{2}$ appears in a straight line with a thickness of $\approx d / 3$.
The mass transfer of a zigzagging bubble $(d=1.5 \mathrm{~mm}$, $v=152 \mathrm{~mm} / \mathrm{s}$ ) is visualized by the PLIF image sequence $(f=300 \mathrm{~Hz})$ shown in Fig. 6 . The bubble oscillates in the out-of-plane direction, and each time it crosses the laser sheet, two spots of dissolved $\mathrm{CO}_{2}$ are visible in the plane. This two-parted structure suggests that the mass transfer is associated with the trailing vortices in the wake of the bubble.

Figure 7 shows a PLIF sequence $(f=500 \mathrm{~Hz})$ featuring a small field of view with a bubble $(d=3.0 \mathrm{~mm}$, $v=199 \mathrm{~mm} / \mathrm{s}$ ) that also oscillates in the out-of-plane
Fig. 6 Sequence of 2D PLIF images showing a bubble with diameter $d=1.5 \mathrm{~mm}$ and rise velocity $v=152 \mathrm{~mm} / \mathrm{s}$ (without Triton X-100)

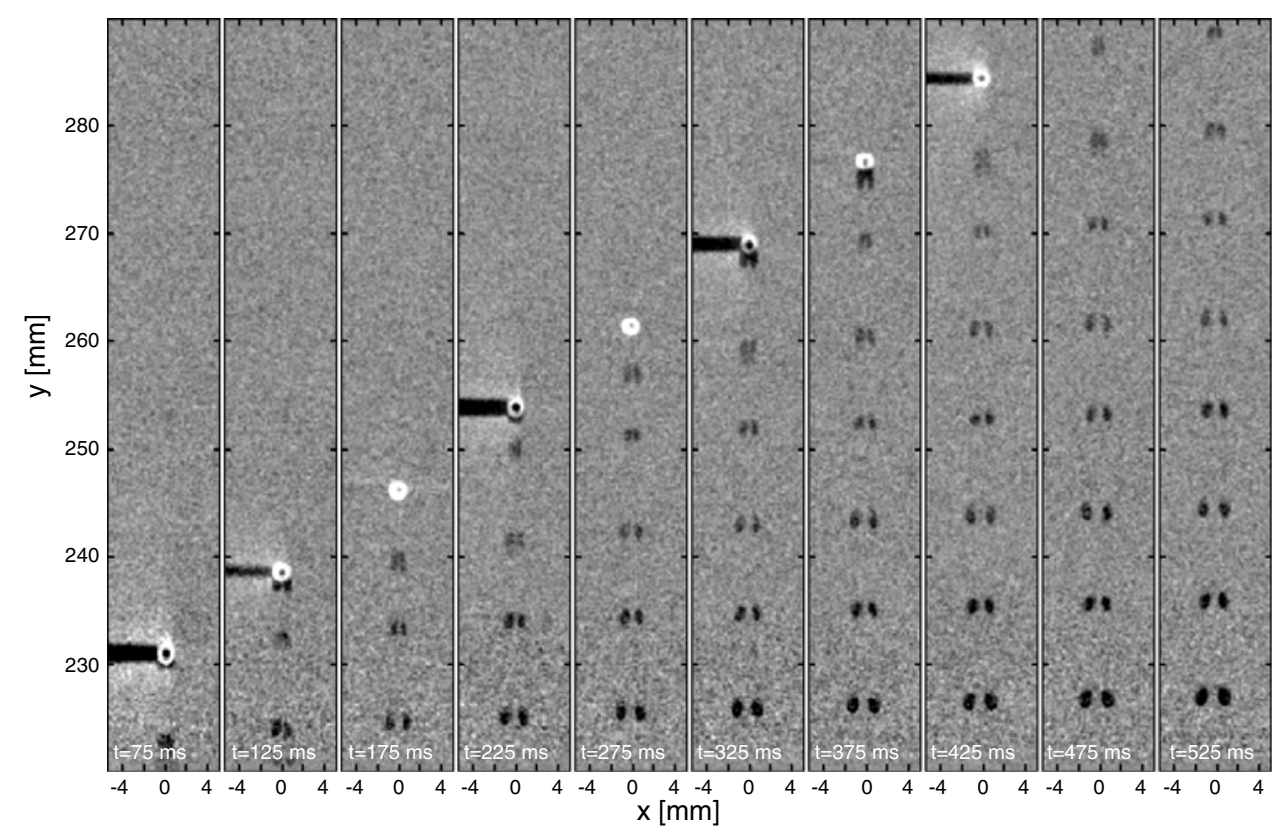


Fig. 7 Sequence of 2D PLIF images showing a bubble with diameter $d=3.0 \mathrm{~mm}$ and rise velocity $v=199 \mathrm{~mm} / \mathrm{s}$ (without Triton X-100). The time between images is a $\Delta t=10 \mathrm{~ms}$ and $\mathbf{b} \Delta t=2 \mathrm{~ms}$ (full temporal resolution)

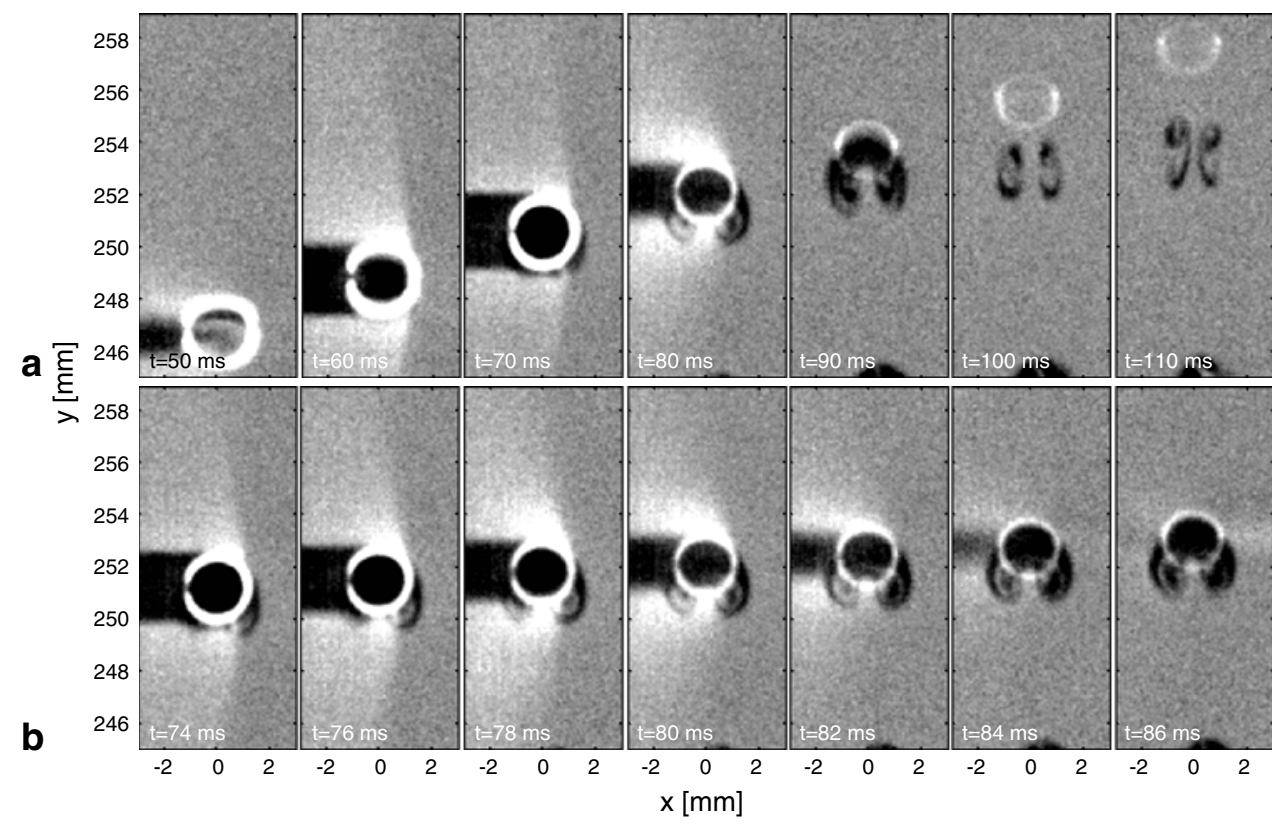

direction. The figure shows every fifth image $(\Delta t=10 \mathrm{~ms}$, upper row) and the full temporal resolution $(\Delta t=2 \mathrm{~ms}$, lower row) of the PLIF sequence. Initially the bubble is rising in front of the laser plane, moving towards it, then crossing the plane ( $t=70 \mathrm{~ms})$ and rising behind it. After the bubble crosses the plane, a symmetric pattern of dissolved $\mathrm{CO}_{2}$ appears in its wake. The curled structure indicates the presence of trailing vortices.

Figure 8 presents a PLIF sequence with a large field of view $\left(46 \times 169 \mathrm{~mm}^{2}\right)$ that was recorded with $f=85 \mathrm{~Hz}$. The bubble $(d=4.4 \mathrm{~mm}, v=242 \mathrm{~mm} / \mathrm{s})$ exhibits significant deformations and oscillates aperiodically in the horizontal plane. The wake of the bubble is characterized by irregular, tortuous structures of dissolved $\mathrm{CO}_{2}$, indicating a turbulent velocity field in the wake. The frequent renewal of liquid in the near wake suggests a high rate of mass transfer.

An example of a 3D PLIF measurement is shown in Fig. 9. The 2D images were recorded with $f=300 \mathrm{~Hz}$ (corresponding to $f=20 \mathrm{~Hz}$ for entire 3D images) and a spacing in out-of-plane direction of $1.5 \mathrm{~mm}$. The 3D image was obtained while the bubble $(d=4.5 \mathrm{~mm}, v=237 \mathrm{~mm} / \mathrm{s})$ leaves the field of view at the upper boundary (visible in the images at $z \leq-7.5 \mathrm{~mm}$ ). According to the discussion in Sect. 1, the 3D images approximate instantaneous concentration fields in the wake region several diameters behind the bubble (corresponding roughly to $y<270 \mathrm{~mm}$ for this example), but not in the near wake $(y>270 \mathrm{~mm})$. While the individual 2D images show disconnected regions of dissolved $\mathrm{CO}_{2}$, the 3D isosurface plot reveals a largescale, continuous meandering trail that consists of smallscale tortuous patterns of $\mathrm{CO}_{2}$.

\section{Summary and conclusions}

A planar laser-induced fluorescence (PLIF) technique for visualization of gas-liquid mass transfer at the interface of rising gas bubbles has been described. It has been shown that $\mathrm{CO}_{2}$ as a tracer gas in an aqueous solution of the $\mathrm{pH}-$ sensitive dye Naphthofluorescein corresponds well with the emission wavelength of a frequency-doubled $\mathrm{Nd}$ :YAG laser. The method features a high spatial resolution and a frame rate of up to $500 \mathrm{~Hz}$, which provides the ability to capture cinematographic image sequences. Using a set of two programmable scanning mirrors for purposes of steering the laser beam, the field of view can be easily adapted to the scale of interest and scanned in the third dimension, which allows for the acquisition of threedimensional LIF image time-series.

The technique has been applied to rising bubbles with diameters between 0.5 and $5 \mathrm{~mm}$ performing rectilinear, oscillatory or irregular motions. The resulting PLIF image sequences reveal the evolution of characteristic structures in the wake of the bubbles and show that the relevant time and length scales can be resolved. The technique thus proves as an extension of the existing experimental methods with the potential of providing new and detailed insights into the spatio-temporal dynamics of mass transfer of rising gas bubbles. The analysis of bubble rise velocities indicates that contaminated conditions are encountered in the Naphthofluorescein solution. While it rules out the investigation of bubbles with non-contaminated interface, this limitation is of minor practical relevance because most technical and natural systems also feature contaminated conditions. 


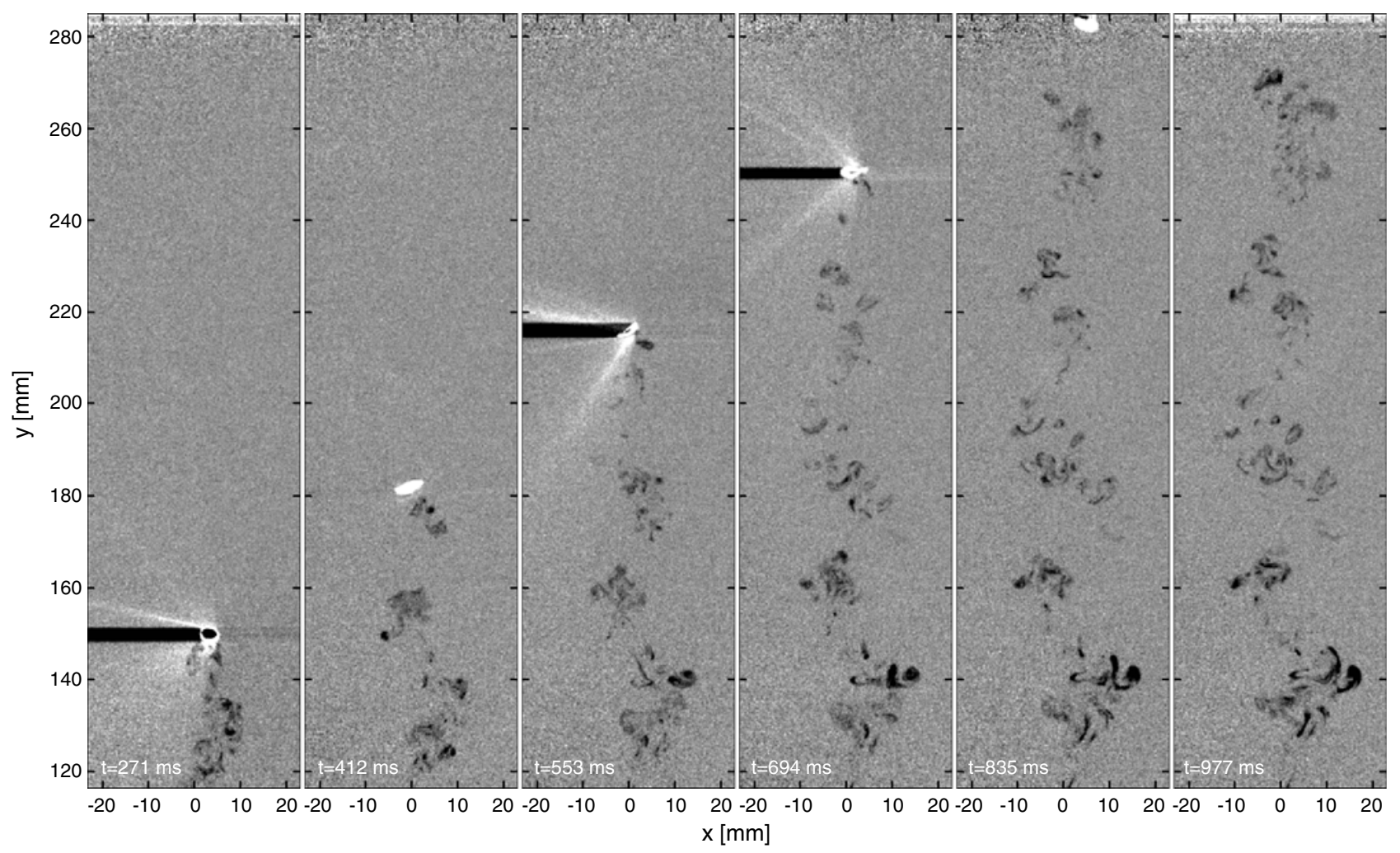

Fig. 8 Sequence of 2D PLIF images showing a bubble with diameter $d=4.4 \mathrm{~mm}$ and rise velocity $v=242 \mathrm{~mm} / \mathrm{s}$ (without Triton X-100)
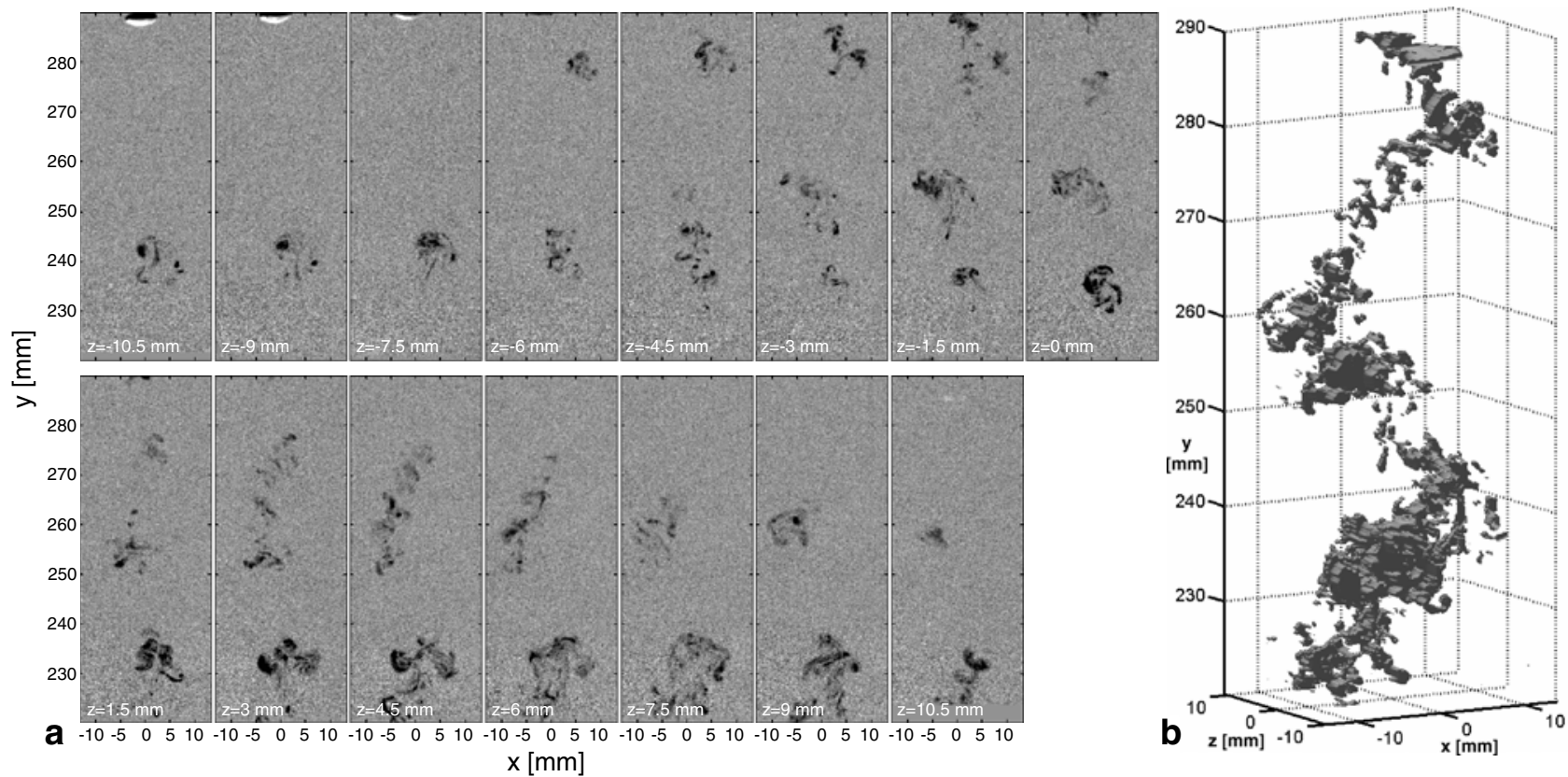

Fig. $93 \mathrm{D}$ PLIF measurement of the wake of a bubble with $d=4.5 \mathrm{~mm}$ and $v=237 \mathrm{~mm} / \mathrm{s}$ (without Triton X-100), represented by a the individual 2D images at different positions in the out-of-plane direction and $\mathbf{b}$ a $3 \mathrm{D}$ isosurface plot 
Open Access This article is distributed under the terms of the Creative Commons Attribution Noncommercial License which permits any noncommercial use, distribution, and reproduction in any medium, provided the original author(s) and source are credited.

\section{References}

Bel Fdhila R, Duineveld PC (1996) The effect of surfactant on the rise of a spherical bubble at high Reynolds and Peclet numbers. Phys Fluids 8(2):310-321

Brücker C (1999) Structure and dynamics of the wake of bubbles and its relevance for bubble interaction. Phys Fluids 11:1781-1796

Clift R, Grace JR, Weber ME (1978) Bubbles, drops and particles. Academic Press, New York

Crimaldi JP (2008) Planar laser induced fluorescence in aqueous flows. Exp Fluids 44:851-863

Deusch S, Dracos T (2001) Time resolved 3D passive scalar concentration-field imaging by laser induced fluorescence (LIF) in moving liquids. Meas Sci Technol 12:188-200

de Vries AWG, Biesheuvel A, van Wijngaarden L (2002) Notes on the path and wake of a gas bubble rising in pure water. Int $\mathrm{J}$ Multiph Flow 28:1823-1835

Ellingsen K, Risso F (2001) On the rise of an ellipsoidal bubble in water: oscillatory paths and liquid-induced velocity. J Fluid Mech 440:235-268

Fujiwara A, Danmoto Y, Hishida K, Maeda M (2004) Bubble deformation and flow structure measured by double shadow images and PIV/LIF. Exp Fluids 36:157-165

Herlina, Jirka GH (2004) Application of LIF to investigate gas transfer near the air-water interface in a grid-stirred tank. Exp Fluids 37:341-349
Jähne B, Haußecker H (1998) Air-water gas exchange. Annu Rev Fluid Mech 30:443-468

Lide DR (2000) CRC handbook of chemistry and physics. CRC Press, Boca Raton

Lindken R, Merzkirch W (2000) Velocity measurements of liquid and gaseous phase for a system of bubbles rising in water. Exp Fluids 29:S194-S201

Lunde K, Perkins RJ (1997) Observations on wakes behind spheroidal bubbles and particles. ASME-FED Summer Meeting, Vancouver, Canada, Paper No. FEDSM97-3530

Magnaudet J, Eames I (2000) The motion of high-reynolds-number bubbles in inhomogeneous flows. Annu Rev Fluid Mech 32:659708

Münsterer T, Jähne B (1998) LIF measurements of concentration profiles in the aqueous mass boundary layer. Exp Fluids 25:190196

Sigma-Aldrich (2008) Naphthofluorescein data sheet. Sigma-Aldrich Corp., St Louis

Tamimi A, Rinker EB, Sandall OC (1994) Diffusion coefficients for hydrogen sulfide, carbon dioxide, and nitrous oxide in water over the temperature range 293-368 K. J Chem Eng Data 39:330-332

Tian X, Roberts PJW (2003) A 3D LIF system for turbulent buoyant jet flows. Exp Fluids 35:636-647

Turney D, Banerjee S (2008) Transport phenomena at interfaces between turbulent fluids. AIChE J 54:344-349

Walker JW, Peirson WL (2008) Measurement of gas transfer across wind-forced wavy air-water interfaces using laser-induced fluorescence. Exp Fluids 44:249-259

Zhang Y, Finch JA (2001) A note on single bubble motion in surfactant solutions. J Fluid Mech 429:63-66 